\title{
Clinical Applications of fMRI
}

Presurgical mapping is the most pressing clinical application for functional MRI (fMRI). Clinical examples have been described elsewhere in the literature (Lee et al., 1999; Thulborn, 1999a). The diagnostic applications in psychiatric disease in which dysfunction has no obvious structural basis are still research-based. An early example is the possible role of fMRI in the diagnosis of probable Alzheimer's disease (Thulborn et al., 2000). Applications in stroke rehabilitation (Booth et al., 1999; Thulborn et al., 1999a), learning disabilities, and neurological disorders remain to be developed. These future clinical applications are likely to require longitudinal examinations and will require the development of calibrated paradigms and a broad clinical experience with each population. These experiences will be inserted into Current Protocols in Magnetic Resonance Imaging as they become pertinent.

The clinical scenario in which fMRI is requested by a referring physician is usually after detection of a lesion in the brain of a patient for whom surgical intervention is being contemplated. The location of eloquent cortex relative to the lesion allows a surgical approach that minimizes loss of cognitive abilities or allows the patient and surgeon to be appropriately prepared to decide on the risk/benefit ratio of the anticipated surgical procedure.

Due to the field-strength dependence of both signal-to-noise performance and the magnetic susceptibility effect of blood oxygenation level dependent (BOLD) contrast, fMRI is best performed on the highest-field strength magnet available (Turner et al., 1993; Thulborn et al., 1996, 1997; Gati et al., 1997). The author's clinical fMRI experience is with both clinical 1.5 and 3.0 T systems (Thulborn et al., 1996, 1999a), although similar results may be expected from other manufacturers if quality assurance (see UNIT A6.2) is adhered to. Because many images are required over a large volume of the brain, multi-planar, echo-planar imaging (Cohen and Weisskoff, 1991) offers a high duty cycle with good image quality. Gradient-echo sequences provide the greatest sensitivity for BOLD contrast and are used routinely (Bandettini et al., 1992). The echo-time $\left(T_{\mathrm{E}}\right)$ is matched to the uncorrected transverse relaxation time $\left(T_{2}^{*}\right)$ in the region of the brain of interest and at the field strength being used ( $\sim 50 \mathrm{msec}$ at $1.5 \mathrm{~T}, 25 \mathrm{msec}$ at $3.0 \mathrm{~T})$. The spatial resolution is dependent on scanner capabilities, but smaller voxels produce greater BOLD contrast by diminishing partial volume effects (Thulborn et al., 1997). Generally, echo-planar images provide voxel dimensions of $3 \times 3 \times 5 \mathrm{~mm}^{3}$ at $1.5 \mathrm{~T}$ and $3 \times 3 \times 3$ $\mathrm{mm}^{3}$ at 3.0 T. The higher signal-to-noise ratio (SNR) at higher field strength allows smaller voxels with the concomitant gain in BOLD contrast.

\section{IMAGING LANGUAGE FUNCTION}

The paradigm for language mapping can take many forms. A block design can be used in which central fixation is compared to visually presented sentences. Each sentence consists of a simple or conjoined sentence followed by a simple question that can be answered as either "TRUE" or "FALSE" by pressing one of two finger switches held in either hand. There are 5 pairs of sentences and questions per $30 \mathrm{sec}$ block, allowing about $6 \mathrm{sec}$ for reading comprehension and answering. As a visual presentation is used, visual acuity must be assured. This is done prior to the imaging session during the demonstration of the task to the patient. Visual acuity issues are addressed with MR-compatible spectacles (MIT Optical). Further concerns about performing the task while in the scanner can be addressed in a scanner simulator (Rosenberg et al., 1997). This also screens for unexpected claustrophobia in a more relaxed situation than in the scanner room, where the time pressure of a busy clinical schedule is more apparent.

Contributed by Keith R. Thulborn and Denise Davis

Current Protocols in Magnetic Resonance Imaging (2001) A6.1.1-A6.1.9

Copyright $\odot 2001$ by John Wiley \& Sons, Inc.

BASIC

PROTOCOL 
The description of the study presented to the patient by the nursing staff and MR technologist during the screening interview and paradigm training is an essential component of establishing rapport with the patient. Such rapport is important to obtain maximum cooperation from the patient. The need for no head motion must be emphasized. This can be aided by encouraging the patient not to talk unless there is a problem. In the same way, during the study in the scanner, the technologist must not ask questions of the patient that require responses, but rather provide information as statements about the progress of the study. Not all patients are good candidates for fMRI, as can be judged from the training session. Patients with difficulty maintaining stationary head position, possibly due to tremors, respiratory difficulties, or pain, or who are not fully attentive, possibly due to medications, should not be considered for this type of study. Other issues that may be contraindications are various metal implants, of which dental braces in children are a good example. These appliances do not represent a hazard to a conventional MR examination, but rather seriously degrade the quality of gradient echo imaging due to magnetic susceptibility effects that can decrease signal intensity across large regions of the brain.

Table A6.1.1 lists the hardware requirements for the BOLD contrast fMRI component of a comprehensive MRI examination. The radiofrequency coil should be a high performance volume coil for whole brain imaging. The gradient set should be capable of single-shot, echo-planar imaging. The typical gradient strength for high quality echo-planar imaging is on the order of $40 \mathrm{mT} / \mathrm{m}$ with slew rates greater than $150 \mathrm{mT} / \mathrm{m} / \mathrm{sec}$. In some cases, there are commercially available portable gradient inserts that can be retrofitted to a scanner to provide such performance, if it is not available from the scanner gradients (Binder et al., 1994). The temporal stability of signal intensity should typically be better than 1\% over 30 min (Thulborn, 1999b). The synchronization control system for paradigm presentation is also useful for simplifying the role of the technologist in synchronizing the paradigm and scanner (Thulborn et al., 1996). The use of a visor helps to minimize head motion (Thulborn, 1999c); however, lower-resolution echo planar imaging (EPI) scans (as described herein) are viable with gradient strengths of $25 \mathrm{mT} / \mathrm{m}$ and slew rates of $75 \mathrm{mT} / \mathrm{m} / \mathrm{sec}$.

NOTE: The eligibility of the patient for fMRI must be considered carefully, and the clinical history must be available to provide an appropriate fMRI protocol. Direct discussion with the referring physician is optimal to minimize failed studies due to poor selection of candidate patients. The cooperation of the patient with cognitive tasks within the scanner can sometimes be gauged from the history. Indications of patient motion should be asked about, including respiratory difficulties, pain, and tremors. Medications can also interfere with and decrease BOLD contrast. Few medications have been evaluated for this potential interference but this should be considered in the interpretation (Gollub et al., 1998). The use of one of the robust primary sensory or motor paradigms serves as

Table A6.1.1 Equipment for fMRI

\begin{tabular}{ll}
\hline Magnet & $\geq 1.5 \mathrm{~T}$ \\
Coil type & Volume transmit/receive head coil \\
Gradient coil strength and slew rate & $\sim 40 \mathrm{mT} / \mathrm{m},>150 \mathrm{mT} / \mathrm{m} / \mathrm{sec}$ \\
Motion cushions & Useful \\
Peripheral gating & Unnecessary except for monitoring \\
Cardiac gating & Unnecessary \\
Respiratory gating & Unnecessary \\
Respirator & Contraindication \\
Oxygen & No \\
Use of contrast agents & No \\
\hline
\end{tabular}

A6.1.2 
the control for such interference. The referring physician can be helpful in emphasizing to the patient the importance of the functional information for surgical planning.

1. Interview the patient to ensure that no contraindications for MRI are present.

Generally standard screening forms are used for all patients scanned in a magnetic resonance system.

The presence of any ferromagnetic metals may be a health hazard to the patient when he or she is inside the magnet, and will also affect the imaging. If in doubt as to the exact composition of the items, it is best to exclude patients with any metal implants; see Shellock (1996) for discussion of what implants may be safely scanned using magnetic resonance.

If a family member is to be in the scan room with the patient, that person must also be screened for contraindications. The presence of a parent can aid studies on a child, but the parent must be instructed to minimize talking to the child during the study.

2. For research studies: Explain the entire procedure to the patient and obtain written informed consent on an appropriate form approved by the Institutional Review Board.

3. Demonstrate the paradigm.

After explaining the difference between a fMRI and conventional MRI examination, the technologist should demonstrate the paradigm to the patient in the interview room. Have the patient perform the paradigm on a computer screen. The technologist should assess the performance of the patient to ensure that the task is appropriate to the skills of the patient. Assess visual acuity at this time, as this situation best matches the scanner distances and screen luminosity.

4. Have the patient change into hospital-supplied clothing after removing all jewelry and mascara. Encourage the use of the bathroom facilities at this time to avoid unnecessary disruption of the scanning schedule.

5. Address any concerns about the scanner environment (e.g., acoustical noise, confinement), preferably in a simulator, in a relaxed manner. Let the patient exert some control over this process by asking questions. Demonstrate the use of ear plugs.

This process is particularly appropriate to gain the cooperation of pediatric patients who are often more curious than anxious.

6. If this study is to be used for stereotaxic localization of a lesion with respect to surface head anatomy within the next $24 \mathrm{hr}$, apply skin markers to the head.

Functional studies are usually done significantly prior to surgery, when such markers would be inappropriate.

7. Take the patient to the scanner, and ask the patient to lie comfortably in supine position on the MR table, positioning in the head radiofrequency coil, thereby replicating the experience in the simulator. With ear plugs, respiratory monitoring, and peripheral monitoring devices in place, stabilize the head with soft cushions, landmarked on the nasion. Place the visor over the face of the patient. Establish the head position with the sighting device of the visor. If stimulation goggles are being used, position the stimulation device so that the patient is comfortable. If only a screen is being used, move the patient into the bore of the magnet and install the screen within the magnet bore or at the end of the patient table.

8. Place the emergency stop device in one of the patient's hands to allow the patient to immediately indicate any major concern during scanning; otherwise, tell the patient to communicate with the technologist over the intercom system if necessary. 
9. Adjust the visual stimulus presentation system for focus by projecting an eye chart. Use the eye chart to center the projected stimulus on the screen.

This step requires verbal interaction with the subject.

IMPORTANT NOTE: Prior to starting the study, the patient should be asked if he or she is comfortable, and then, if so, told to maintain the current position. Unnecessary talking is to be discouraged due to increased head motion that results. During the fMRI study, the technologist should avoid asking questions of the subject but rather make statements that inform but do not require verbal responses from the patient (see above).

If the patient is unable to follow the procedure up to this stage, they may not be an appropriate candidate for fMRI. The technologist should then proceed with the remainder of the conventional MRI examination.

\section{Sequence 1: Rapid sagittal scout view}

10. Optional: Some very high-field scanners $(3 \mathrm{~T})$ now have first- and second-order shim gradients with appropriate rapid shimming software to improve the magnetic field homogeneity over the head of each patient. This can be used as a separate and initial acquisition if available.

11. Use a sagittal scout view for graphic prescription of the functional imaging plane in transverse, coronal, or oblique planes (Table A6.1.2). If higher-order shimming is not available, this sequence also performs a first order autoshim to improve magnet homogeneity for the specific patient under study.

Table A6.1.2 describes typical acquisition parameters.

This acquisition should be of sufficient quality for recognizing anatomic landmarks rather than of minimal duration.

Table A6.1.2 Parameters for Sagittal Acout Image at 1.5 and 3.0 T

\begin{tabular}{ll}
\hline Patient position & Supine \\
Scan type & Spin echo \\
Imaging plane (orientation) & Sagittal \\
Central slice or volume center & Lateral canthus of the eye \\
Echo time $\left(T_{\mathrm{E}}\right)$ & Minimum full (or $16 \mathrm{msec}$ at \\
& $1.5 \mathrm{~T}, 14 \mathrm{msec}$ at $3.0 \mathrm{~T})$ \\
Repeat time $\left(T_{\mathrm{R}}\right)$ & $500 \mathrm{msec}$ at $1.5 \mathrm{~T}, 500-700 \mathrm{msec}$ \\
& at $3.0 \mathrm{~T}$ \\
Flip angle $(\mathrm{FA})$ & $90^{\circ}$ \\
Field of view $(\mathrm{FOV}, \mathrm{FOV}$ & \\
Resolution $(\Delta x, \Delta y)$ & $240 \mathrm{~mm}, 240 \mathrm{~mm}$ \\
Number of data points collected $\left(N_{\mathrm{x}}, N_{\mathrm{y}}\right)$ & $0.94 \mathrm{~mm}, 1.25 \mathrm{~mm}$ \\
Display matrix $\left(D_{\mathrm{x}}, D_{\mathrm{y}}\right)$ & 256,192 \\
Slice thickness $(\Delta z)$ & 256,256 \\
Number of slices & $5 \mathrm{~mm}$ \\
Slice gap & 12 \\
Number of acquisitions $\left(N_{\mathrm{acq}}\right)$ & $1 \mathrm{~mm}$ \\
Swap read and phase encoding & 1 \\
Slice locations & No \\
Saturation pulses & L36-R36 \\
Scan time & None \\
& $1 \mathrm{~min}, 26 \mathrm{sec}$ at $1.5 \mathrm{~T}, 2 \mathrm{~min}, 14$ \\
& $\mathrm{sec}$ at $3.0 \mathrm{~T}$ \\
\hline &
\end{tabular}


Ultra-fast imaging after automated shimming and reference scan for ghost correction form the basis for the functional study. Acquisition parameters will vary with scanner and operating system, but Table A6.1.3 shows typical parameters.

IMPORTANT NOTE: The beginning of the paradigm presentation and the image acquisition must be synchronized. This is most easily achieved with a synchronization control system (Thulborn et al., 1996).

The duration of the acquisition is based on experience with patients and paradigms. 10 min is the upper limit of patient tolerance before head motion and fatigue counterbalance improved statistical power. The rule of thumb is that at least 60 images per paradigm condition are required to reach statistically reliable results for the language paradigm described above, in most cooperative patients. The images for this paradigm are best performed in the transverse plane and should cover the vertex of the brain to the bottom of the temporal lobes. As fMRI becomes more routine, the current restrictions of duty cycle and total number of images that can be acquired will be removed by the scanner vendors. Specific pulse sequences to be used for fMRI are undergoing rapid development and will be quickly outdated. For these reasons, specific sequences will not be given.

\section{Sequence 3: High-resolution anatomical images}

13. Table A6.1.4 shows typical acquisition parameters for a high-resolution dataset showing the surface topography.

As functional activation maps are of low spatial resolution, a high-resolution dataset can be useful to the neurosurgeon to plan a surgical approach to a lesion avoiding not only eloquent cortex, but also structures such as veins and arteries. Although there are well known differences in image distortions between echo-planar and conventional images, these distortions are quantifiable and should be considered in the surgical planning (Cohen, 1999).

Table A6.1.3 Parameters for $\mathrm{fMRI}$ at 1.5 and 3.0 T

\begin{tabular}{ll}
\hline Patient position & Supine \\
Scan type & Gradient echo echo-planar \\
Imaging plane (orientation) & Transverse \\
Central slice or volume center & Lateral canthus of the eye \\
Echo time $\left(T_{\mathrm{E}}\right)$ & $50 \mathrm{msec}$ at $1.5 \mathrm{~T}, 25-50 \mathrm{msec}$ at \\
& $3.0 \mathrm{~T}$ \\
Repeat time $\left(T_{\mathrm{R}}\right)$ & $3000 \mathrm{msec}$ \\
Flip angle $(\mathrm{FA})$ & $90^{\circ}$ \\
Field of view $\left(\mathrm{FOV}, \mathrm{\textrm {x }}, \mathrm{FOV}_{\mathrm{y}}\right)$ & $200 \mathrm{~mm}, 200 \mathrm{~mm}$ \\
Resolution $(\Delta x, \Delta y)$ & $3.1 \mathrm{~mm}, 3.1 \mathrm{~mm}$ \\
Number of data points collected $\left(N_{\mathrm{x}}, N_{\mathrm{y}}\right)$ & $64,64(\mathrm{ramp} \mathrm{sampling})$ \\
Display matrix $\left(D_{\mathrm{x}}, D_{\mathrm{y}}\right)$ & 64,64 \\
Slice thickness $(\Delta z)$ & $5 \mathrm{~mm}$ at $1.5 \mathrm{~T}, 3 \mathrm{~mm}$ at $3.0 \mathrm{~T}$ \\
Number of slices & 12 \\
Slice gap & $1 \mathrm{~mm}$ \\
Number of acquisitions $\left(N_{\mathrm{acq}}\right)$ & 1 \\
Swap read and phase encoding & Yes \\
Read direction & Left-right \\
Slice locations & Graphic prescription, 12 slices \\
Saturation pulses & None \\
Scan time & $6 \mathrm{~min}$ for 6 cycles of $1 \mathrm{~min}$ each \\
\hline
\end{tabular}


Table A6.1.4 Parameters for High-Resolution Anatomic Images at 1.5 and 3.0 T

\begin{tabular}{ll}
\hline Patient position & Supine \\
Scan type & 3-D Spoiled Gradient Echo \\
Imaging plane (orientation) & Transverse \\
Central slice or volume center & Lateral canthus of the eye \\
Echo time $\left(T_{\mathrm{E}}\right)$ & Minimum $(\sim 5 \mathrm{msec})$ \\
Repeat time $\left(T_{\mathrm{R}}\right)$ & $25 \mathrm{msec}$ \\
Flip angle $(\mathrm{FA})$ & $40^{\circ}$ \\
Fields of view $\left(\mathrm{FOV}, \mathrm{FOV}_{\mathrm{y}}\right)$ & $240 \mathrm{~mm}, 240 \mathrm{rmm}$, with $r=3 / 4$ \\
& $($ rectangular field of view) \\
Resolution $(\Delta x, \Delta y)$ & $0.94 \mathrm{~mm}, 1.25 \mathrm{~mm}$ \\
Number of data points collected $\left(N_{\mathrm{x}}, N_{\mathrm{y}}\right)$ & $256,192 r$, with $r=3 / 4$ (rectangular \\
& field of view) \\
Display matrix $\left(D_{\mathrm{x}}, D_{\mathrm{y}}\right)$ & 256,256 \\
Slice thickness $(\Delta z)$ & $1.5 \mathrm{~mm}$ \\
Number of slices & 124 \\
Slice gap & $0 \mathrm{~mm}$ \\
Number of acquisitions $\left(N_{\mathrm{acq}}\right)$ & 1 \\
Swap read and phase encoding & No \\
Read direction & Anterior-posterior \\
Slice locations & Graphic prescription, 124 slices \\
Saturation pulses & None \\
Scan time & 7 min, 44 sec \\
\hline
\end{tabular}

This high-resolution study allows the anterior and posterior commissures to be identified and covers the entire brain so that Talairach coordinates (Talairach and Tournoux, 1988) can be used if requested.

IMPORTANT NOTE: Following an fMRI study, further sequences would be expected to involve the conventional imaging to fully characterize the nature of the lesion for which surgical planning is being done. The identification of large blood vessels is useful to exclude artifacts from veins in activation maps. The acquisition of a cerebral venogram can be useful for this purpose. If contrast is to be given to characterize the lesion, this represents an opportunity to perform a perfusion study to establish the regions of normal perfusion in which BOLD contrast can be reliably interpreted. Some perfusion studies are described in Chapter A1.

\section{COMMENTARY}

\section{Background Information}

Functional imaging to map eloquent cortex is still in its infancy for clinical applications. The clinical requirement for a high success rate on individual patients with a comprehensible timely report is a challenge for any neuroradiology service. Success depends on careful quality assurance at all stages including patient preparation, paradigm selection, data acquisition, and image analysis. Quality assurance and paradigm design are treated in detail as separate units.

The statistical processing of fMRI data can be performed on some clinical scanners. In some cases, the activation map can be observed in real time during the acquisition. The processing is a simple $t$-test for a two-condition paradigm as used for the language paradigm discussed above. Otherwise, the data must be moved off the scanner into a computer environment in which other public domain or commercial software can be used to process the data. Many statistical methods have been discussed (Lange, 1999) and some have been made available as software packages (Gold et al., 1998). These packages usually allow the activation maps colored by statistical significance to be superimposed over the high-resolution anatomic images (Cox, 1996).

For clinical decisions based on fMRI results, the areas of activation must be reported with respect to the location of the lesion. The Talairach coordinates for areas of activation are less useful to the surgeon than distances and direc- 
tions from the lesion and readily identified topographic landmarks. The positions of large veins that may complicate the interpretation of BOLD contrast (especially at $1.5 \mathrm{~T}$ but less important at 3.0 T; Thulborn et al., 1999b) are useful to indicate to the physician.

As registration errors can occur due to patient motion and image artifacts, an estimate of these potential errors should be included.

For lesions that may perturb the hemodynamic coupling of neuronal activity, care should be taken to ensure that the BOLD contrast remains valid up to the edge of the lesion. Confidence in this assumption may be improved by performing additional studies such as diffusion and perfusion MR studies as described in Chapter A1. In regions in which diffusion or perfusion measurements are abnormal, it is best to assume that BOLD contrast will be less sensitive and that no interpretation of function can be made reliably. This has not proven to be a significant problem in clinical settings of arteriovenous malformations, tumors, or even subacute stroke; however, one recent case report emphasizes the concern for this potential pitfall (Holodny et al., 1999).

There is significant variation in the extent of activation between different individuals. This is not surprising given the wide variation observed with behavioral measurements of cognition; however the patterns of activation presumably reflecting the large-scale neurocognitive networks involved in cognitive processing seem to be reproducible (Mesulam, 1990, 1998). Knowledge of the nature of this network in an appropriately matched control population is helpful for clear interpretation of activation maps in individual patients.

\section{Critical Parameters and Troubleshooting}

When an activation study fails to yield the expected results, a systematic approach to understanding the source of the problem can be informative and help improve subsequent studies. If all the images have been obtained and used in the analysis without a satisfactory result then the following steps may be helpful.

1. Daily quality assurance (QA) should be verified to ensure that scanner performance and concomitant image quality has not degraded. If image quality is below specifications, a service call may be required. This is usually easily checked and the least likely source of any problems. The patient study should not be done until daily QA has been verified.
2. Patient cooperation in terms of motion can be verified by viewing the images from the functional study in an animation loop. Rapid head movements of greater than one voxel are readily apparent as are slow drifts in head position during the study. Head motion is most often the source of failure even in apparently cooperative patients. This problem is reduced by comfortable positioning, head support, and the visor (Thulborn, 1999c).

3 . Cooperation with performing the paradigm is also essential. The language paradigm responses should be verified to have high accuracy and normal response times. Low accuracy and fast responses may indicate guessing (e.g., incorrect strategy, low motivation, inappropriate match to patient skills). The control tasks should show good activation maps as patient cooperation with the task is virtually assured by definition.

4. Determine the time between the patient entering the scanner and the attempt at the failed fMRI study. Loss of alertness after prolonged times in the scanner does not improve activation maps.

5. Ensure that the technologist or nurse has informed the patient appropriately and in detail about the nature of the study and that the patient has understood the instructions. A new technologist may not have developed the same skill set in establishing patient rapport as experienced MRI staff. A formal educational program for the staff may be required.

6. Data management and analysis can sometimes lead to errors, especially if the procedures are not used routinely. Practice and experience result in efficiently performed studies with optimal results. A trial data set can be used to train new or recalibrate previous operator skills when necessary.

\section{Anticipated Results}

An activation map in which the areas of significant BOLD contrast are displayed in color over the detailed anatomic images including the lesion of concern is the desirable result. Images through the entire brain would be best to show all anatomic landmarks for the surgeon. The addition of markers if the patient is going immediately to the operation would help anatomic localization. This implies that the analysis is available within minutes and that the data can be transmitted immediately to the operating room for use on stereotaxically guided surgical instrumentation. With well characterized paradigms and experience with 


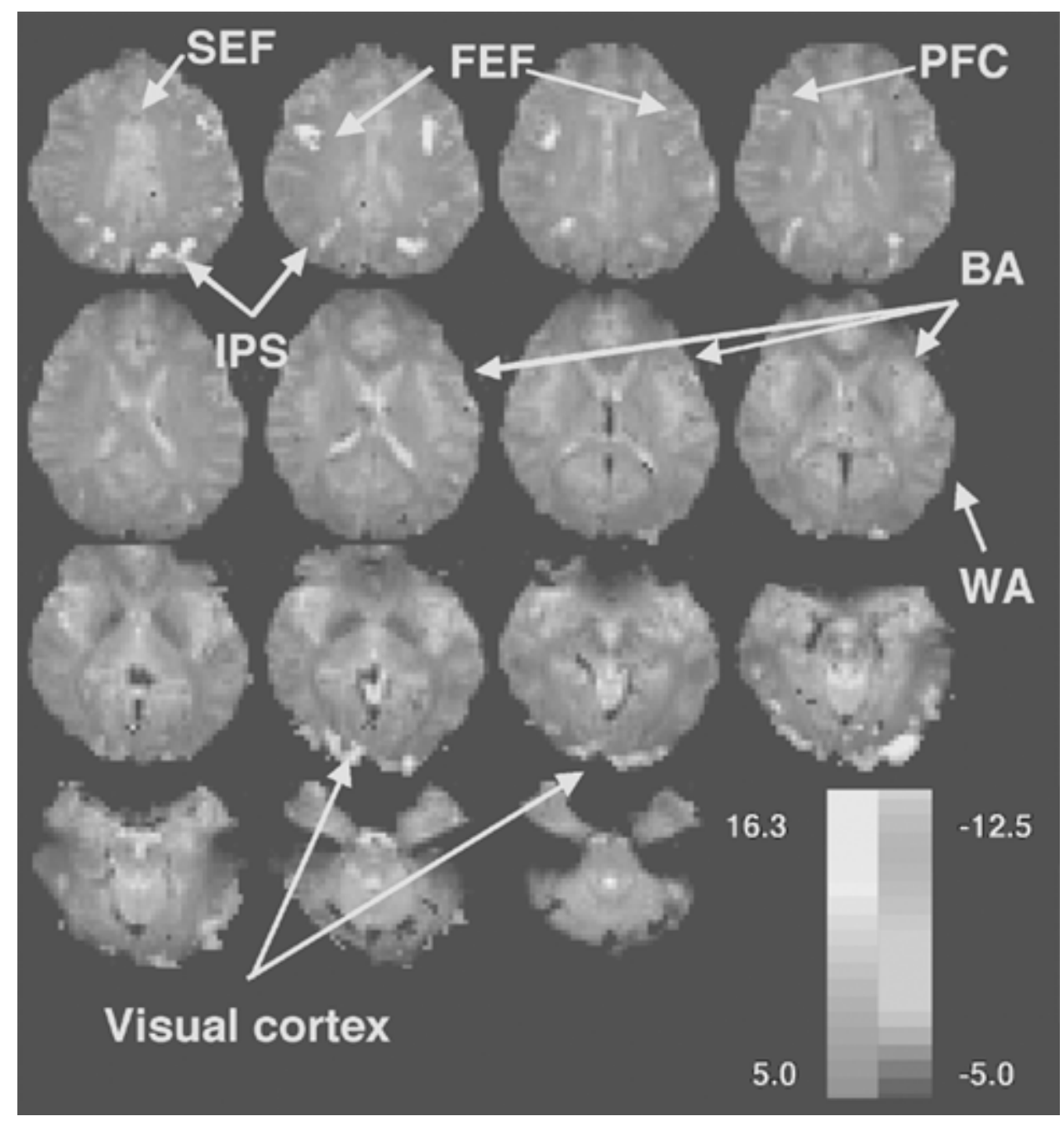

Figure A6.1.1 Representative activation map at 3.0 T for language paradigm, showing extensive network activation with areas of frontal eye fields (FEF, precentral sulcus), supplementary eye fields (SEF, medial frontal cortex), prefrontal cortex (PFC), intraparietal sulcus (IPS), Broca's area (BA, left inferior frontal cortex), Wernicke's area (WA, left superior temporal gyrus), and visual cortex (calcarine sulcus). This pattern is observed in adults irrespective of gender or handedness. The right inferior frontal cortex also shows activation in the contralateral location to Broca's area as can sometimes be observed with Wernicke's area. The color scale indicates the $t$-test statistic for activation. The higher $t$-statistic (yellow) has greater statistical significance. See color plate.

interpretation of such data, these results are achievable at a high success rate with appropriately chosen patients. The analysis of perfusion and diffusion data should be included in the report to indicate in which areas of the brain a BOLD contrast interpretation can be confidently rendered. Images for a normal individual performing the language paradigm are shown in Figure 6.1.1.

\section{Literature Cited}

Bandettini, P.A., Wong, E.C., Hinks, R.S., Tikofsky, R.S., and Hyde, J.S. 1992. Time course EPI of human brain function during task activation. Magn. Reson. Med. 25:390-397.
Binder, J.R., Rao, S.M., Hammeke, T.A., Yetkin, F.Z., Jesmanowicz, A., Bandettini, P.A., Wong, E.C., Estowski, L.D., Goldstein, M.D., Haughton, V.M., and Hyde, J.S. 1994. Functional magnetic resonance imaging of human auditory cortex. Annu. Neurol. 35:662-672.

Booth, J.R., MacWhinney, B., Thulborn, K.R., Sacco, K., Voyvodic, J., and Feldman, H.M. 1999. Functional organization of activation patterns in children: Whole brain fMRI imaging during three different cognitive tasks. Prog. Neuro-Psychopharmacol. Biol. Psychiat. 23:669-682.

Cohen, M.S. 1999. Echo-planar imaging and functional MRI. In Medical Radiology: Diagnostic Imaging and Radiation Oncology. Functional 
MRI. (C. Moonen and P. Bandettini., eds.) pp. 137-148. Springer-Verlag, Berlin.

Cohen, M.S. and Weisskoff, R.M. 1991. Ultrafast imaging. Magn. Reson. Imaging 9:1-37.

Cox, R.W. 1996. AFNI, software for analysis and visualization of functional magnetic resonance neuroimages. Comput. Biomed. Res. 29:162173.

Gati, J.S., Menon, R.S., Ugurbil, K., and Rutt, B.K. 1997. Experimental determination of the BOLD field strength dependence in vessels and tissue. Magn. Reson. Med. 38:296-302.

Gold, S., Christian, B., Arndt, S., Zeien, G., Cizadlo, T., Johnson, D.L., Fiaum, M., and Andreasen, N.C. 1998. Functional MRI statistical software packages: A comparative analysis. Hum. Brain Mapp. 6:73-84.

Gollub, R.L., Breiter, H.C., Kantor, H., Kennedy, D., Gastfriend, D., Mathew, R.T., Makris, N., Guimaraes, A., Riorden, J., Campbell, T., Foley, M., Hyman, S., Rosen, B., and Weisskoff, R.. 1998. Cocaine decreases cortical cerebral blood flow but does not obscure regional activation in functional magnetic resonance imaging in human subjects. J. Cereb. Blood. Flow Metab. 18:724734.

Holodny, A.I., Schulder, M., Lui, W.C., Maldjian, J.A., and Kalnin, A.J. 1999. Decreased BOLD functional MR activation of the motor and sensory cortices adjacent to a glioblastoma multiforme: Implications for image-guided neurosurgery. Am. J. Neuroradiol. 20:609-612.

Lange, N. 1999. Statistical procedures for functional MRI. In Medical Radiology: Diagnostic Imaging and Radiation Oncology, Functional MRI. (C. Moonen and P. Bandettini., eds.) pp. 301335. Springer-Verlag, Berlin.

Lee, C.C., Ward, H.A., Sharbrough, F.S., Meyer, F.B., March, W.R., Raffel, C., So, E.L., Cascino, G.D., Shin, C., Xu, Y., Riederer, S.J., and Jack, C.R. 1999. Assessment of functional MR imaging in neurosurgical planning. Am. J. Neuroradiol. 20:1511-1519.

Mesulam, M.-M. 1990. Large-scale neurocognitive networks and distributed processing for attention, language and memory. Annu. Neurol. 28:597-613.

Mesulam, M.-M. 1998. From sensation to cognition. Brain 121:1013-1052.

Rosenberg, D.R., Sweeney, J.A., Gillen, J.S., Chang, S.Y., Varanelli, M.J., O'Hearn, K., Erb, P.A., Davis, D., and Thulborn, K.R. 1997. Magnetic resonance imaging of children without sedation: Preparation with simulation. J. Am. Acad. Child Adolesc. Psychiatry 36:853-59

Shellock, F.G. 1996. Pocket Guide to MR Procedures and Metallic Objects. Lippincott-Raven, Philadelphia.
Talairach, J. and Tournoux, P. 1988. Co-planar stereotaxic atlas of the human brain 3D proportional system: An approach to cerebral imaging. (G. Thieme, ed.) Springer-Verlag, New York.

Thulborn, K.R. 1999a. Clinical rationale for very high field (3.0 Tesla) functional MR imaging. Topics Magn. Reson. Imaging 10:37-50.

Thulborn, K.R. 1999b. Quality assurance in clinical and research echo-planar functional MRI. In Medical Radiology: Diagnostic Imaging and Radiation Oncology, Functional MRI. (C. Moonen and P. Bandettini, eds.) pp. 337-345. SpringerVerlag, Berlin.

Thulborn, K.R. 1999c. Visual feedback to stabilize head position for fMRI. Magn. Reson. Med. 41:1039-1043.

Thulborn, K.R., Davis, D., Erb, P., Strojwas, M., and Sweeney, J.A. 1996. Clinical fMRI: Implementation and experience. NeuroImage 4:S101S107.

Thulborn, K.R., Chang, S.Y., Shen, G.X., and Voyvodic, J.T. 1997. High resolution echo-planar fMRI of human visual cortex at 3.0 Tesla. NMR Biomed. 10:183-90.

Thulborn, K.R., Carpenter, P.A., and Just, M.A. 1999a. Plasticity of language-related brain function during recovery from stroke. Stroke 30:749754.

Thulborn, K.R., Gindin, T., and Talagala, S.L. 1999b. High-resolution echo-planar fMRI measures localized cortical microvascular responses, not just large venous drainage patterns. In Proceedings Seventh Scientific Meeting International Society of Magnetic Resonance in Medicine. Philadelphia, PA, May 27.

Thulborn, K.R., Martin, C., and Voyvodic, J. 2000. fMRI using a visually guided saccade paradigm in Alzheimer's disease. Am. J. Neuroradiol. 21:524-531.

Turner, R., Jezzard, P., Wen, H., Kwong, K.K., Bihan, D.L., Zeffiro, T. and Balaban, R.S. 1993. Functional mapping of the human visual cortex at 4 and 1.5 Tesla using deoxygenation contrast EPI. Magn. Reson. Med. 29:277-279.

\section{Key Reference}

Moonen, C. and Bandettini, P. (eds.) 1999. Medical Radiology: Diagnostic Imaging and Radiation Oncology, Functional MRI. Springer-Verlag, Berlin.

Comprehensive book on all issues related to fMRI

Contributed by Keith R. Thulborn and

Denise Davis

University of Illinois at Chicago

Chicago, Illinois 